\title{
System-environment correlations and Markovian embedding of quantum non-Markovian dynamics
}

\author{
Steve Campbell, ${ }^{1}$ Francesco Ciccarello, ${ }^{2,3}$ G. Massimo Palma, ${ }^{2,3}$ and Bassano Vacchini ${ }^{4,1}$ \\ ${ }^{1}$ Istituto Nazionale di Fisica Nucleare, Sezione di Milano, via Celoria 16, 20133 Milan, Italy \\ ${ }^{2}$ Università degli Studi di Palermo, Dipartimento di Fisica e Chimica, via Archirafi 36, I-90123 Palermo, Italia \\ ${ }^{3}$ NEST, Istituto Nanoscienze-CNR, 56127 Pisa, Italy \\ ${ }^{4}$ Dipartimento di Fisica “Aldo Pontremoli”, Università degli Studi di Milano, via Celoria 16, 20133 Milan, Italy
}

(Received 28 May 2018; published 30 July 2018)

\begin{abstract}
We study the dynamics of a quantum system whose interaction with an environment is described by a collision model, i.e., the open dynamics is modeled through sequences of unitary interactions between the system and the individual constituents of the environment, termed "ancillas," which are subsequently traced out. In this setting, non-Markovianity is introduced by allowing for additional unitary interactions between the ancillas. For this model, we identify the relevant system-environment correlations that lead to a non-Markovian evolution. Through an equivalent picture of the open dynamics, we introduce the notion of "memory depth" where these correlations are established between the system and a suitably sized memory rendering the overall system+memory evolution Markovian. We extend our analysis to show that while most system-environment correlations are irrelevant for the dynamical characterization of the process, they generally play an important role in the thermodynamic description. Finally, we show that under an energy-preserving system-environment interaction, a nonmonotonic time behavior of the heat flux serves as an indicator of non-Markovian behavior.
\end{abstract}

DOI: 10.1103/PhysRevA.98.012142

\section{INTRODUCTION}

The inherent fragility of pure quantum states necessitates that we develop a firm understanding of how these systems interact with their environments. If the system under scrutiny is weakly coupled to the environment and/or the latter has negligible correlation time, the ensuing dynamics can be well approximated using Markovian master equations [1]. These typically rely on the assumption that the environment remains unaffected by its coupling to the system, thereby yielding negligible system-reservoir correlations. Such an approximation generally needs to be relaxed in the description of nonMarkovian dynamics. Equally important with understanding the underlying mechanisms that govern the dynamics of an open quantum system is assessing their thermodynamic principles [2]. Advances in this regard have been made for both Markovian [3-7] and, to a lesser extent, non-Markovian [8-13] dynamics.

Of course, not all dynamics fall within the memoryless environment paradigm and, as such, there have been rapid developments in tools to model quantum non-Markovian dynamics [14-20]. Within the plethora of approaches, collision models (CMs) stand out. In its simplest memoryless version, a $\mathrm{CM}$ assumes that the reservoir consists of a large number of initially uncorrelated subunits or ancillas with which the open system collides one at a time [21-24]. Suitable modifications endow this basic $\mathrm{CM}$ with memory, making it an advantageous tool to tackle quantum non-Markovian dynamics [25-38]. In addition, CMs have found application in quantum optics [39-42], quantum gravity [43,44], quantum control [45-48], and quantum thermodynamics [49-61].

Remarkably, the discrete nature of CMs together with their tractability allow one to study how the progressive inclusion of system-reservoir correlations introduces non-
Markovianity in the open dynamics. Along this line, McCloskey and Paternostro [62] considered a non-Markovian $\mathrm{CM}$ where the system-ancilla collisions are interspersed with nearest-neighbor ancilla-ancilla (AA) collisions, where the latter ones introduce a memory mechanism. They investigated the effect of erasing correlations between $S$ (the system) and the last collided ancilla or between $S$ and the next-to-last ancilla, showing that the latter erasure scheme results in increased non-Markovianity.

In the CM of Ref. [62], the dynamical map of $S$, and thus the degree of non-Markovianity, will no longer change if correlations with ancillas prior to the next-to-last one are retained. This is because only nearest-neighbor ancillas collide: once a given ancilla has collided with $S$ and then with the next ancilla, it can no longer affect the dynamics of $S$. In fact, only correlations between the system and the portion of environment with which it is currently interacting matter, with the size of such environment's portion being related to the range of intrabath interactions. Based on this, one may ask whether, by embedding $S$ into an extended open system $\mathfrak{S}$ that comprises a small subset of environmental ancillas as well, correlations between such a redefined open system and the remaining environment can now be neglected without affecting the dynamics of $\mathfrak{S}$, and hence of $S$. Such an effective description of a non-Markovian dynamics is sometimes referred to as Markovian embedding [30,63]. According to this picture, it is reasonable to expect that the number of ancillas in $\mathfrak{S}$ should reflect the size of the bath portion whose correlations with $S$ cannot be neglected.

With the main goal of assessing and formalizing the above picture, in this work we explore the connection between system-environment correlations and Markovian embedding. We focus on a non-Markovian quantum CM featuring AA 
collisions without necessarily restricting to nearest-neighbor AA collisions and with no constraints on the form of the pairwise coupling Hamiltonian ruling both system-ancilla and AA collisions (many CMs including Ref. [62] focus on partialSWAP collisions). If $d$ is the range of interancillary collisions (i.e., each ancilla collides with the next $d$ ancillas only), then correlations between $S$ and the last $d$ ancillas it collided with cannot be erased, prior to the intraenvironment AA collisions, without affecting the open dynamics of $S$. This observation will motivate us to introduce within this framework the concept of memory depth as measured by the integer $d$. It will be shown that a Markovian embedding for $S$ holds provided it is incorporated into a composite system $\mathfrak{S}$ featuring, in addition, as many ancillas as the memory depth $d$.

While only correlations with a limited portion of the bath are essential to capture the open dynamics, generally all system-environment correlations are instead essential for the thermodynamical properties. In the last part of the work, we will provide evidence of this by considering the decomposition of the system's entropy change in terms of reversible and irreversible entropy production, with the latter being, in particular, dependent on system-environment correlations.

The remainder of the paper is organized as follows. In Sec. II, we introduce the collision model that we will use. In Sec. III, we briefly describe how quantities such as the degree of non-Markovianity and correlations will be measured throughout our analysis. In Sec. IV, we explicitly examine the relevance of correlations in a nearest-neighbor CM, recapitulating and extending some known results. In Sec. V, we establish equivalent descriptions of the model in terms of a Markovian embedding and introduce the notion of memory depth. Section VI studies the thermodynamical aspects of the model and, in Sec. VII, we conclude and discuss the generality of our results and their possible application to other non-Markovian models.

\section{SYSTEM-ENVIRONMENT MODEL}

The CM that we consider in this work belongs to the class of non-Markovian CMs where the reservoir's memory mechanism is due to the occurrence of AA collisions [30,31,62,64,65], the first of which was introduced in Ref. [28] and stimulated a number of studies on a new class of nonMarkovian dynamics [66-71].

The $\mathrm{CM}$ assumes that the reservoir or environment $E$ is made up of a large number of identical ancillas, each called $E_{n}$. The total state of the system and environment is initially factorized, i.e.,

$$
\rho_{S E}(0)=\rho_{S}(0) \otimes \rho_{E_{1}} \otimes \rho_{E_{2}} \otimes \cdots,
$$

with the initial correlations between $S$ and any ancilla as well as between any two ancillas thus being zero.

The dynamics proceeds through pairwise interaction processes or "collisions" between $S$ and a portion of the environment, $\left\{E_{n}, \ldots E_{n+d-1}\right\}$. In what follows, we assume these collisions happen successively; however, our results hold for simultaneous interactions. These are followed by intraenvironment pairwise collisions among $E_{n}, E_{n+1}, \ldots, E_{n+d}$, with $d$ the interancillary collision range (later on reinterpreted as memory depth). For $d=1$, corresponding to nearest-neighbor

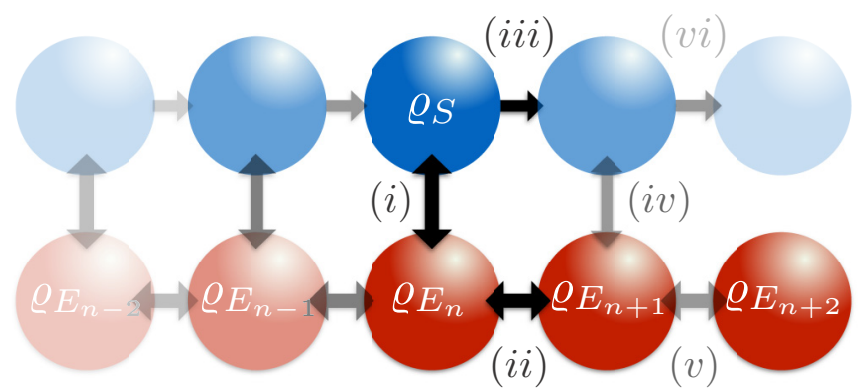

FIG. 1. Schematic of the collision model for nearest-neighbor AA collisions (i.e., $d=1$ ). In the $n$th step of the dynamics $(i), S$ collides with $E_{n}$ and next ( $\left.i i\right) E_{n}$ collides with $E_{n+1}$. The system $S$ then moves forward (iii). At the $(n+1)$ th step (iv), $S$ collides with $E_{n+1}$ and (v) $E_{n+1}$ collides with $E_{n+2}$. For the case of $d$-range AA collisions, after $S$ has interacted with the ancillas $E_{n}, \ldots, E_{n+d-1}$, AA collisions up to range $d$ take place between the ancillas $E_{n}, \ldots, E_{n+d}$.

AA collisions, $S$ thus initially collides with $E_{1}$, after which $E_{1}$ collides with $E_{2}$, then $S$ collides with $E_{2}$, and $E_{2}$ with $E_{3}$, and so on. All collisions are assumed to be unitary. In Fig. 1, we show a schematic of the CM considered.

If AA collisions are removed, the CM reduces to a fully memoryless one: prior to collision $S-E_{n}$, ancilla $E_{n}$ is still in its initial state and thus fully uncorrelated with $S$, and hence past history cannot affect the dynamics. The occurrence of AA collisions instead endows the environment with memory: if $d=1$ (to fix the ideas) after colliding with $S$, ancilla $E_{n-1}$ undergoes an AA collision with $E_{n}$; thereby, $S$ and $E_{n}$ are already correlated before colliding with each other. An analogous argument holds for $d>1$.

We will assume throughout that $S$ and each ancilla $E_{n}$ are qubits with free Hamiltonians, $\hat{H}_{S}=-\omega_{0} \sigma_{S z}$ and $\hat{H}_{E_{n}}=-$ $\omega_{0} \sigma_{E_{n} z}$, respectively, with $\sigma_{i=x, y, z}$ being the usual Pauli operators (we set $\hbar=1$ ).

The general interaction Hamiltonian describing a collision reads

$$
\hat{H}_{i j}=-\frac{1}{2}\left(J_{x} \sigma_{i x} \sigma_{j x}+J_{y} \sigma_{i y} \sigma_{j y}+J_{z} \sigma_{i z} \sigma_{j z}\right)
$$

(tensor product symbols are not shown), where, for systemancilla collisions, $i$ and $j$ stand for the system $S$ and an ancilla, respectively, while for AA collisions, $i$ and $j$ correspond to two different ancillas with $i \neq j$.

We define a "step" as the sequence of collisions

$$
\begin{gathered}
\hat{U}_{1}=\prod_{i=1}^{d} e^{-i \hat{H}_{S E_{i}} \tau}, \\
\hat{U}_{n>1}=\left(\prod_{i=(n-1) d+1}^{n d} e^{-i \hat{H}_{S E_{i}} \tau}\right)\left(\prod_{l, m=(n-2) d+1}^{(n-1) d+1} e^{-i \hat{H}_{E_{l} E_{m}} \tau}\right),
\end{gathered}
$$

for $l<m$ and where $\tau$ is the collision time, and we have allowed for arbitrarily ordered pairwise AA interactions (in what follows, for brevity we will denote the action of the AA interactions with $\left.\mathcal{V}_{[\cdot]}\right)$. The evolution operator of the overall system (i.e., $S+E$ ) at step $n$ is thus given by the composition $\hat{U}_{n} \cdots \hat{U}_{1}$.

For $J_{x}=J_{y}=J_{z}=J$, the interaction Hamiltonian (2) is energy preserving and the corresponding unitary is the 
partial-SWAP operation $\hat{U}=\cos (J \tau) \mathbb{1}-i \sin (J \tau) \hat{S}$ often considered in the CM literature [51,52,62,64]. Here, angle $J \tau$ (defined such that $0 \leqslant J \tau \leqslant \frac{\pi}{2}$ ) measures the strength of AA collisions: these have zero and maximum effect if, respectively, $J \tau=0$ and $J \tau=\frac{\pi}{2}$. In the former case, the model reduces to a fully Markovian one. In the latter case (corresponding to perfect swap), a strongly non-Markovian dynamics occurs instead since $S$ behaves as if it is interacting with the same ancilla at all times $[28,62]$.

\section{NON-MARKOVIANITY AND CORRELATIONS}

To quantify the degree of non-Markovianity of the open dynamics of $S$, we will use the criterion underpinning the widely adopted Breuer-Laine-Piilo (BLP) measure [16], according to which non-Markovian behavior is associated with a backflow of information from the environment to the system. The occurrence of information backflow depends on the time behavior of the trace distance between two different initial states of $S$, namely,

$$
\mathcal{D}_{n}=\frac{1}{2}\left\|\rho_{S n}^{(1)}-\rho_{S n}^{(2)}\right\|_{1}
$$

where $\|\cdots\|_{1}$ is the trace norm [72] while $\rho_{S n}^{(I)}$ is the $n$ th-step state of $S$ when it starts in $\rho_{S}^{(I)}(0)$. Thus, in the present $\mathrm{CM}$ framework, time is a discrete variable. Under Markovian dynamics, $\mathcal{D}$ monotonically decreases with time, whatever the initial pair of states $\left\{\rho_{S}^{(1)}(0), \rho_{S}^{(2)}(0)\right\}$. Thereby, a nonmonotonic behavior of this quantity for at least one pair of initial states is sufficient to conclude that the governing dynamics is nonMarkovian [16]. However, we remark that this is not a necessary condition as there may be orthogonal pairs resulting in a monotonic decay of the trace distance $\mathcal{D}_{n}$, while the dynamics, nevertheless, are non-Markovian. It can be shown [73] that the pair of initial states yielding maximum deviation from a monotonic decay of the trace distance is orthogonal and lies on the boundary of the state space (i.e., the Bloch sphere in the present case of qubits). This underpins our choice of the specific initial pairs of states that we will focus on in our analysis.

A major focus of this work is the relevance of systemenvironment correlations. Specifically, in terms of the CM introduced in Sec. II, we will be concerned with bipartite correlations between $S$ and a single environmental ancilla $E_{i}$. These can be quantified through the quantum mutual information (MI) [72] for the state $\rho_{S E_{i}}$

$$
I_{S E_{i}}\left(\rho_{S E_{i}}\right)=S\left(\rho_{S}\right)+S\left(\rho_{E_{i}}\right)-S\left(\rho_{S E_{i}}\right),
$$

where $S(\rho)=-\operatorname{Tr}[\rho \log \rho]$ is the von Neumann entropy [72], while $\rho_{S}=\operatorname{Tr}_{E_{i}} \rho_{S E_{i}}$ is the reduced state of $S$ ( $\rho_{E_{i}}$ is defined analogously). It is widely accepted that the mutual information captures the full amount of correlations, both classical and quantum. For product states $\rho_{S E_{i}}=\rho_{S} \otimes \rho_{E_{i}}$, the mutual information always vanishes. In the general case, however, $\rho_{S} \otimes \rho_{E_{i}}$ does not equal $\rho_{S E_{i}}$ and the mutual information is nonzero. Based on this, we see that in the general case, the replacement $\rho_{S E_{i}} \rightarrow \rho_{S} \otimes \rho_{E_{i}}$ (which is a quantum map) extracts the uncorrelated part of the joint state $\rho_{S E_{i}}$ and, as such, erases the $S-E_{i}$ correlations altogether.

At this point, we highlight an important observation. It is worth recalling that the knowledge of $\rho_{S}=\operatorname{Tr}_{E_{i}} \rho_{S E_{i}}$ is sufficient to determine the final state of $S, \rho_{S}^{\prime}$, after a quantum map on $S$ (unitary or not), $\mathcal{M}_{S} \otimes \mathcal{I}_{E_{i}}$, is applied on $\rho_{S E_{i}}$. In such cases, thereby, if one is interested in the state of $S$ only, erasing correlations, i.e., applying the map $\rho_{S E_{i}} \rightarrow \rho_{S} \otimes \rho_{E_{i}}$, has no effect.

\section{RELEVANT CORRELATIONS: OPEN DYNAMICS}

It is instructive for our purposes to see how the last property discussed in the previous section applies to a memoryless CM (namely, the CM of Fig. 1 in the absence of AA collisions). Before colliding with $E_{n}, S$ is correlated with all previous ancillas. Yet, only its reduced state right before the collision with $E_{n}$ is needed to work out the open dynamics. This means that correlations between $S$ and each ancilla can be erased after they have collided without affecting the open dynamics of $S$, which in practice means it is enough to keep track of the state of $S$ only throughout (that is, only one qubit).

In line with Ref. [62], the approach that we adopt to investigate the importance of correlations is studying how the repeated erasure of system-ancilla correlations affects the open dynamics of $S$, in particular, non-Markovianity of the system's evolution and its thermodynamical properties. Specifically, we consider erasing correlations according to the following three schemes:

(i) Scheme A: The correlations established after $S$ has collided with $E_{n}$ are erased before $E_{n}$ interacts with $E_{n+1}$.

(ii) Scheme B: The correlations established after $S$ has collided with $E_{n}$ are erased only after $E_{n}$ has collided with $E_{n+1}$, but before $S$ collides with $E_{n+1}$.

(iii) Scheme C: The correlations established after $S$ has collided with $E_{n}$ are retained, like scheme B, during the $E_{n}-E_{n+1}$ collision and, at variance with scheme B, even during the $S-E_{n+1}$ collision. Afterward, they are erased before $E_{n+1}$ collides with $E_{n+2}$.

In practice, the above means that in addition to the state of $S$, we keep track of the state of one environment ancilla (scheme A), two ancillas (scheme B), and three ancillas (scheme C). Following from the last property outlined in Sec. III, the dynamics for schemes B and C will necessarily be identical; however, we explicitly consider this case as it will be instructive when assessing the thermodynamics of the CM in Sec. VI. Schemes A and B are precisely those considered in Ref. [62] where all interactions were a partial SWAP.

In Figs. 2(a) and 2(b), we examine the behavior of the trace distance, given by Eq. (5), for different orthogonal pairs of the system's initial state. We have (arbitrarily) assumed all environmental qubits are initialized in their ground states and consider $J_{x}=2 J_{y}=J_{z}$. In Fig. 2(a), we see that when the correlations are erased before the intraenvironment interactions take place, i.e., scheme A, the trace distance $\mathcal{D}(t)$ [cf. (5)] exhibits a nontrivial, yet monotonically decaying behavior for both the initial pairs $\{| \pm\rangle\}$ and $\{|0\rangle,|1\rangle\}$. However, the less invasive correlation's erasure prescribed by scheme B leads to an increase of non-Markovian behavior [cf. solid curves in Fig. 2(b)]. We confirm that the dynamics are unaffected when more environmental ancillas are retained following scheme $\mathrm{C}$. As evidenced by the dashed curves in Fig. 2(b), the resulting trace distance is invariant and it is easy to confirm that the dynamical state of the system is identical under schemes 
(a)

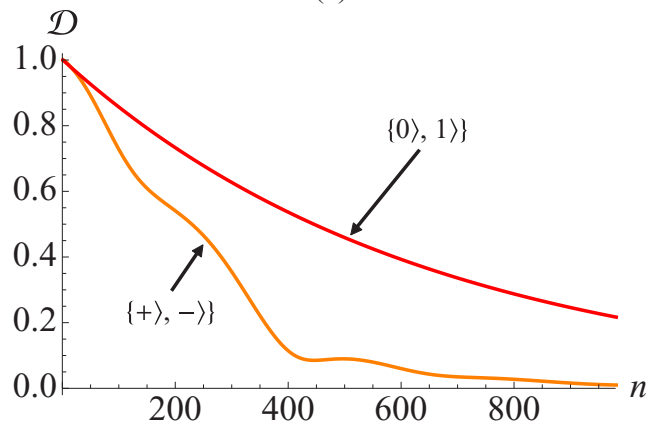

(b)

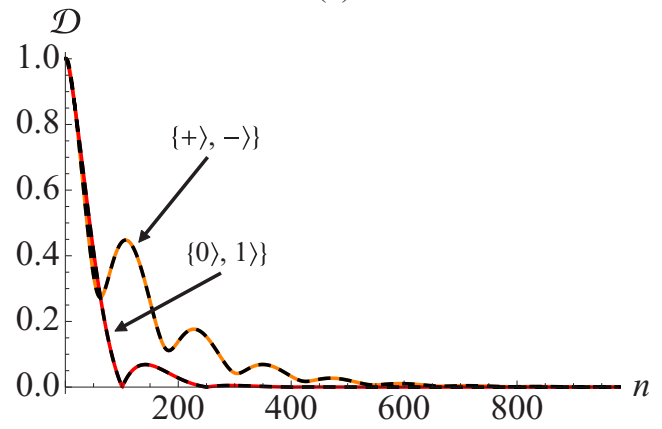

FIG. 2. (a),(b) Trace distance $\mathcal{D}$ against the number of steps for two representative pairs of initial orthogonal pure states of $S$ taken to be $\{|0\rangle,|1\rangle\}$ and $\{| \pm\rangle\}$, with $| \pm\rangle=(|0\rangle \pm|1\rangle) / \sqrt{2}$ following erasure (a) scheme A and (b) schemes B (solid colors) and C (dashed black line). Note that in (b) the curves are identical between the two schemes $\mathrm{B}$ and $\mathrm{C}$, indicating the irrelevance of the additionally retained correlations. We set $J_{x}=2 J_{y}=J_{z}=1$ [cf. Eq. (2)] for both system-ancilla and AA collisions and $J \tau_{S A}=0.05, J \tau_{\mathrm{AA}}=0.95 \frac{\pi}{2}$ [cf. (4)]. In both panels, each ancilla is initialized in $|0\rangle_{n}$.

$\mathrm{B}$ and $\mathrm{C}$. It follows that retaining any more environmental subunits has no effect on the resulting dynamics of the system. One can compare these figures with those of Ref. [62] to assess the dependence of the non-Markovianity on the details of the interactions involved. In passing, note that the nonMarkovianity of the dynamics, as captured by revivals in the trace distance, is significantly diminished by changing the interaction model. This can be understood considering that the partial-SWAP operation used in Ref. [62] is the most "memory-mimicking" interaction one can use and thus leads to the strongest exhibition of non-Markovian features.

It should be stressed that the invariance of the dynamics between schemes $\mathrm{B}$ and $\mathrm{C}$ is notwithstanding the establishment of correlations between $S$ and all previous ancillas, in particular $E_{n-1}$. In Fig. 3(a), we show that there are nonzero correlations shared between the system and ancillas $E_{n}$ (dashed black line) and $E_{n-1}$ (solid red line) before the $(n+1)$-th collision. Notice that the $S-E_{n-1}$ correlations are erased under scheme B while they are retained under scheme $\mathrm{C}$, and despite this difference the overall evolution of $S$ remains unaffected. This confirms that only certain correlations are important in dictating the dynamical features, and therefore non-Markovian nature, of the system's evolution. In the considered setting, where the environment is restricted to nearest-neighbor interactions, the correlations shared between the system $S$ and environment (a)

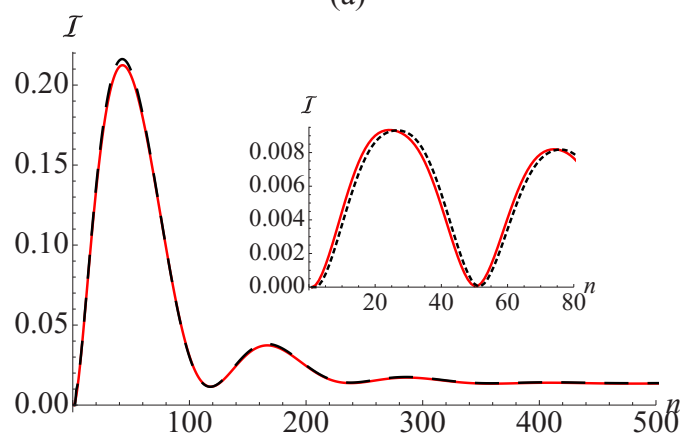

(b)

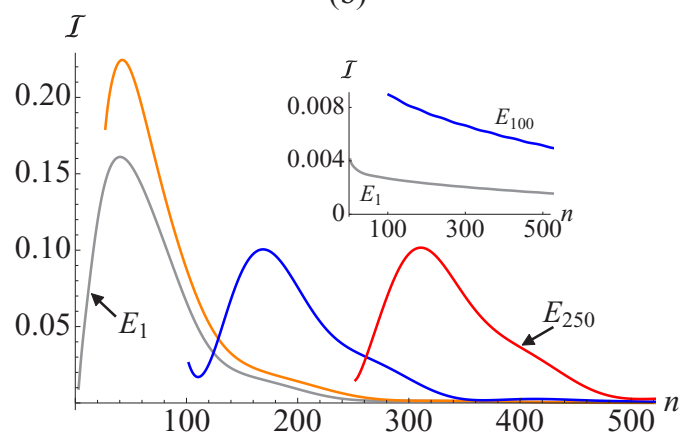

FIG. 3. (a) Quantum mutual information, given by Eq. (6), for scheme $\mathrm{C}$ between $S$ and $E_{n}$ (dashed black line) and $E_{n-1}$ (solid red line) for the same parameters as Fig. 2(b) and with the system initialized in $|+\rangle$. Inset: For comparison, we also show the same quantities when the partial-SWAP operation is used for all collisions, as done in Ref. [62]. (b) Quantum mutual information shared between the system and a fixed environment ancilla $E_{1}, E_{25}, E_{100}$, and $E_{250}$ (curves from left to right), against number of steps $n$, setting $J_{x}=$ $2 J_{y}=J_{z}=1$ and initializing the system in $|+\rangle$. Inset: Same as for the main panel, except switching off the AA interaction so that the dynamics is fully Markovian. In all plots, each ancilla is initialized in its ground state.

ancillas $E_{n}$ and $E_{n+1}$ completely characterize the open dynamics. All other correlations, i.e., those shared between $S$ and $E_{i}$ for $n>i \geqslant 1$, are irrelevant. This can be seen from Fig. 3(b), where we fix an ancilla and assess the MI shared between it and the system during the ensuing dynamics. Clearly there are correlations present long after the ancilla has interacted with the system, but such correlations do not affect the open dynamics. Since the ancilla state no longer changes after all its interactions are over, the change in the MI is entirely due to changes in the state of $S$. This behavior is robust to the particular details defining the interactions, as shown in the insets where we compute the same quantities when all interactions are a partial SWAP [Fig. 3(a)] and even for the Markovian limit when the AA collisions are switched off [Fig. 3(b)].

As we will show by means of an analytic argument in Sec. V, the general features shown in Figs. 2 and 3 are generic; see in particular Eqs. (12) and (14). Furthermore, in the analytic treatment, we also consider interactions beyond nearest neighbor, involving multiple systems, showing that this can be a natural way to introduce a hierarchy of memory effects and the notion of a memory depth. 


\section{MARKOVIAN EMBEDDING AND MEMORY DEPTH}

We next provide a general framework supporting an effective description of the open dynamics that, in particular, better clarifies the relevance of system-environment correlations and, most importantly, shows the effectiveness of a Markovian embedding of the open system under study. Both aspects will be linked to the characteristic range of intraenvironment interactions (here embodied by the range of AA collisions $d$ ), which will be reinterpreted as the memory depth. Such an effective description holds regardless of dimensionality and type of coupling of all the involved particles.

We begin by addressing the $d=1$ case (nearest-neighbor AA collisions). Let us first introduce a suitable notation. The Hilbert spaces of $S$ and the $n$th environmental ancilla are called $\mathcal{H}_{S}$ and $\mathcal{H}_{E_{n}}$, respectively. We call $\mathcal{U}_{S E_{n}}[\cdot]=\hat{U}_{S E_{n}} \cdot \hat{U}_{S E_{n}}^{\dagger}$ the unitary map describing the $S-E_{n}$ collision and $\mathcal{V}_{E_{n+1} E_{n}}[\cdot]=$ $\hat{V}_{E_{n+1}, E_{n}} \cdot \hat{V}_{E_{n+1}, E_{n}}^{\dagger}$ the unitary map describing the $E_{n}-E_{n+1}$ AA collision.

Consider the joint Hilbert space of $S$ and $E_{n}, \mathcal{H}_{S} \otimes \mathcal{H}_{E_{n}}$, and let $\mathcal{T}\left(\mathcal{H}_{S} \otimes \mathcal{H}_{E_{n}}\right)$ be the set of all physical states therein. We will consider a collection of completely positive tracepreserving (CPTP) maps, i.e., maps which ensure the states are physical throughout the evolution [1,72], parametrized by the discrete index $n$,

$$
\Phi_{n}: \mathcal{T}\left(\mathcal{H}_{S} \otimes \mathcal{H}_{E_{n-1}}\right) \rightarrow \mathcal{T}\left(\mathcal{H}_{S} \otimes \mathcal{H}_{E_{n}}\right),
$$

which sends states in $\mathcal{T}\left(\mathcal{H}_{S} \otimes \mathcal{H}_{E_{n-1}}\right)$ to states in $\mathcal{T}\left(\mathcal{H}_{S} \otimes\right.$ $\left.\mathcal{H}_{E_{n}}\right)$, defined as

$$
\begin{gathered}
\Phi_{1}[w]=\mathcal{U}_{S E_{1}}[w], \\
\Phi_{n>1}[w]=\operatorname{Tr}_{E_{n-1}} \mathcal{U}_{S E_{n}} \circ \mathcal{V}_{E_{n} E_{n-1}}\left[w \otimes \rho_{E_{n}}\right],
\end{gathered}
$$

where $w$ stands for an arbitrary state of $S$ and $E_{n-1}$. Here, $\rho_{E_{n}}$ is the initial state of ancilla $E_{n}$ [recall that the system and ancillas start in the factorized state (1)].

We can next introduce a map that returns at step $n$ the joint state of $S$ and the last ancilla which $S$ collided with as

$$
\Phi(n)[w]=\Phi_{n} \circ \ldots \circ \Phi_{1}[w],
$$

such that $\rho_{S E_{1}}(1)=\Phi(1)\left[\rho_{S} \otimes \rho_{E_{1}}\right], \rho_{S E_{2}}(2)=\Phi(2)\left[\rho_{S E_{1}}\right]$, and so on. Map $\Phi(n)$ is manifestly memoryless, according to virtually all Markovianity criteria proposed in the literature [14], since it results from the composition of CPTP maps and trivially fulfills $\Phi(n)=\Phi(n-m) \circ \Phi(m)$ for any $1 \leqslant m<n$.

We also define the dynamical map of $S$ as $\rho_{S}(n)=$ $\Lambda(n)\left[\rho_{S}(0)\right]$, which returns the evolved state of $S$ at each step $n$ for any given initial state $\rho_{S}(0)$. Clearly, if $\Phi(n)$ is known, then so is $\Lambda(n)$ once a partial trace over the last collided ancilla is taken,

$$
\rho_{S}(n)=\Lambda(n)\left[\rho_{S}(0)\right]=\operatorname{Tr}_{E_{n}} \Phi(n)\left[\rho_{S}(0) \otimes \rho_{E_{1}}\right] .
$$

Unlike $\Phi(n)$, the dynamical map $\Lambda(n)$ is, in general, nonMarkovian (for instance, in the case that AA collisions are full swaps; see end of Sec. II). It is, however, natural to define this open dynamics as first-order Markovian, in the sense that by embedding $S$ into a larger system $\mathfrak{S}$ that is comprised of only one additional environmental ancilla, such a redefined open system $\mathfrak{S}$ undergoes a fully Markovian dynamics described by map $\Phi(n)$ [cf. Eq. (9)]. This terminology is introduced by analogy with the corresponding notion in classical stochastic processes [74].

A stronger memory (in the above sense), called secondorder Markovian accordingly, arises for $d=2$ in which case AA collisions involving three, rather than two, ancillas occur. As before, the overall dynamics results from the application of maps describing system-ancilla and AA collisions, the latter now described by a tripartite unitary map $\mathcal{V}_{E_{n+2} E_{n+1} E_{n}}$. The analog of $\Phi_{n}$ [cf. Eqs. (7) and (8)] now sends physical states of $\mathcal{H}_{S} \otimes \mathcal{H}_{E_{n-3}} \otimes \mathcal{H}_{E_{n-2}}$ to states of $\mathcal{H}_{S} \otimes \mathcal{H}_{E_{n-1}} \otimes \mathcal{H}_{E_{n}}$, and is defined as

$$
\begin{aligned}
\Phi_{1}^{(2)}[w]= & \mathcal{U}_{S E_{2}} \mathcal{U}_{S E_{1}}[w] \\
\Phi_{n>1}^{(2)}[w]= & \operatorname{Tr}_{E_{2 n-2} E_{2 n-3}} \mathcal{U}_{S E_{2 n}} \mathcal{U}_{S E_{2 n-1}} \\
& \circ \mathcal{V}_{E_{2 n-1} E_{2 n-2} E_{2 n-3}}\left[w \otimes \rho_{E_{2 n-1}} \otimes \rho_{E_{2 n}}\right],
\end{aligned}
$$

where $w$ now stands for a state in the tripartite Hilbert space $\mathcal{H}_{S} \otimes \mathcal{H}_{E_{2 n-3}} \otimes \mathcal{H}_{E_{2 n-2}}$.

In analogy with Eq. (9), the associated map that returns the joint state of $S$ and the last two collided ancillas is given by

$$
\Phi^{(2)}(n)[w]=\Phi_{n}^{(2)} \circ \ldots \circ \Phi_{1}^{(2)}[w],
$$

such that we obtain $\rho_{S E_{1} E_{2}}(1)=\Phi^{(2)}(1)\left[\rho_{S} \otimes \rho_{E_{1}} \otimes \rho_{E_{2}}\right]$, $\rho_{S E_{3} E_{4}}(2)=\Phi^{(2)}(2)\left[\rho_{S E_{1} E_{2}}\right]$, and so on. Like map (9), this is still fully Markovian again because it results from the composition of CPTP maps. The dynamical map of $S$ is obtained from this as [cf. Eq. (10)]

$$
\rho_{S}(n)=\Lambda^{(2)}(n)\left[\rho_{S}\right]=\operatorname{Tr}_{E_{2 n} E_{2 n-1}} \Phi^{(2)}(n)\left[\rho_{S} \otimes \rho_{E_{1}} \otimes \rho_{E_{2}}\right] \text {. }
$$

The obtained transformations $\left\{\Lambda^{(2)}(n)\right\}$ describe a discrete dynamics which can be naturally termed second-order Markovian since it goes over to a Markovian dynamics by enlarging the description to include two environmental ancillas. This notion can naturally be extended by considering reduced dynamics with an arbitrary memory depth $d$.

The direct connection between memory depth and range of intraenvironmental interactions can easily be seen considering the following equivalent representation for the first-and second-order Markovian maps:

$$
\begin{aligned}
& \Lambda^{(1)}(n)\left[\rho_{S}\right]=\operatorname{Tr}_{E_{n} \ldots E_{1}} \mathcal{U}_{S E_{n}} \cdots \\
& \mathcal{U}_{S E_{2}} \mathcal{V}_{E_{2} E_{1}} \mathcal{U}_{S E_{1}}\left[\rho_{S} \otimes \bigotimes_{i=1}^{n} \rho_{E_{i}}\right] \text {, } \\
& \Lambda^{(2)}(n)\left[\rho_{S}\right]=\operatorname{Tr}_{E_{2 n} \ldots E_{1}} \mathcal{U}_{S E_{2 n}} \mathcal{U}_{S E_{2 n-1}} \cdots \mathcal{U}_{S E_{4}} \mathcal{U}_{S E_{3}} \mathcal{V}_{E_{3} E_{2} E_{1}} \\
& \times \mathcal{U}_{S E_{2}} \mathcal{U}_{S E_{1}}\left[\rho_{S} \otimes \bigotimes_{i=1}^{2 n} \rho_{E_{i}}\right] \text {. }
\end{aligned}
$$

It should be noted that the study of memory effects due to interactions involving higher orders of subunits has been considered by Çakmak et al. in Ref. [64], where it has been shown that indeed this can lead to a higher degree of nonMarkovianity according to recently introduced measures [16].

\section{Effective description via Markovian embedding}

Let us first consider the simplest situation where the dynamics can be characterized as first-order Markovian such that the 


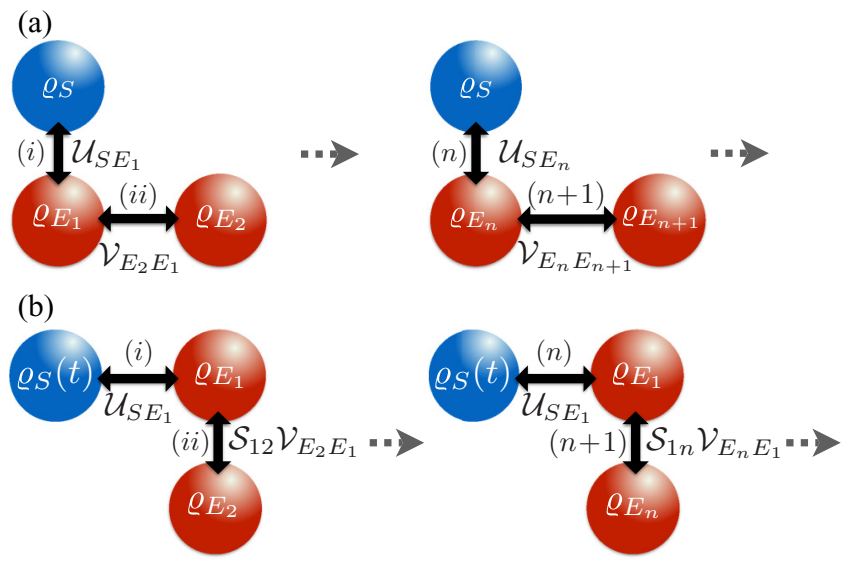

FIG. 4. Schematics of the different equivalent representations of the open dynamics of $S$ in the CM of Fig. 1. (a) The system $S$ collides sequentially with the different environmental ancillas, which in turn interact afterward among themselves. (b) The system always interacts with the same ancilla (working as a memory), which in turn sequentially collides with the other ancillas.

evolved system state after $n$ steps is given by Eq. (12). Due to the fact that the initial state is fully factorized and interactions only take place in a pairwise fashion, the transformation can be arranged in a nested form,

$$
\begin{aligned}
\rho_{S}(n)= & \Lambda(n)\left[\rho_{S}\right] \\
= & \operatorname{Tr}_{E_{n}} \mathcal{U}_{S E_{n}}\left[\operatorname{Tr}_{E_{n-1}} \mathcal{V}_{E_{n} E_{n-1}} \mathcal{U}_{S E_{n-1}}\right. \\
& \times\left[\cdots \operatorname { T r } _ { E _ { 2 } } \mathcal { V } _ { E _ { 3 } E _ { 2 } } \mathcal { U } _ { S E _ { 2 } } \left[\operatorname{Tr}_{E_{1}} \mathcal{V}_{E_{2} E_{1}} \mathcal{U}_{S E_{1}}\right.\right. \\
& \left.\left.\left.\times\left[\rho_{S} \otimes \rho_{E_{1}}\right] \otimes \rho_{E_{2}}\right] \cdots\right] \otimes \rho_{E_{n}}\right]
\end{aligned}
$$

This scheme can be pictorially described through the schematic in Fig. 4(a). Equation (14) shows that in order to fully describe the open dynamics of $S$, it suffices to deal with only three qubits at each step ( $S$ and two ancillas), despite that correlations are being generated between $S$ and all the ancillas which $S$ collided with. In particular, the erasure scheme A (see Sec. IV) corresponds to replacing, in Eq. (14), $\mathcal{U}_{S E_{1}}\left[\rho_{S} \otimes \rho_{E_{1}}\right]$ with the product of the first marginal with the initial state of the ancillas, i.e., $\operatorname{Tr}_{E_{1}}\left(\mathcal{U}_{S E_{1}}\left[\rho_{S} \otimes \rho_{E_{1}}\right]\right) \otimes \rho_{E_{1}}$ (an analogous replacement being made at subsequent steps). Scheme B in Sec. IV instead corresponds precisely to the scheme in Eq. (14), while scheme $\mathrm{C}$ is recovered by postponing the partial trace by one step. To rigorously introduce the notion of memory depth, an alternative formulation can be considered, which puts into evidence the existence of a memory ancilla mediating the interaction between system and environment, and connects the memory depth to the size of the memory ancilla.

Let us first introduce the unitary swap map $\mathcal{S}_{1,2}$, which exchanges the state of two systems (having the same dimension) as $\mathcal{S}_{1,2}\left[\rho_{1} \otimes \rho_{2}\right]=\hat{S}_{1,2} \rho_{1} \otimes \rho_{2} \hat{S}_{1,2}=\rho_{2} \otimes \rho_{1}$, with $\hat{S}_{1,2} \equiv \hat{S}_{1,2}^{\dagger}$ the standard swap operator. This fulfills the basic properties

$$
\operatorname{Tr}_{E_{2} E_{1}} \mathcal{S}_{1,2}[\cdots]=\operatorname{Tr}_{E_{2} E_{1}}[\cdots]
$$

and $\mathcal{S}_{1,2} \circ \mathcal{V}_{E_{2} E_{1}}=\mathcal{V}_{E_{1} E_{2}} \circ \mathcal{S}_{1,2}$. The latter identity, if $E_{2}$ is replaced with an arbitrary ancilla $E_{m}$, can be written more generally as

$$
\mathcal{S}_{1, m} \circ \mathcal{V}_{E_{n} E_{m}}=\mathcal{V}_{E_{n} E_{1}} \circ \mathcal{S}_{1, m} .
$$

Consider now the reduced system dynamics defined by

$$
\begin{aligned}
\rho_{S}^{\prime}(n)= & \operatorname{Tr}_{E_{n} \ldots E_{1}} \mathcal{U}_{S E_{1}} \mathcal{S}_{1, n} \mathcal{V}_{E_{n} E_{1}} \mathcal{U}_{S E_{1}} \cdots \\
& \mathcal{S}_{1,3} \mathcal{V}_{E_{3} E_{1}} \mathcal{U}_{S E_{1}} \mathcal{S}_{1,2} \mathcal{V}_{E_{2} E_{1}} \mathcal{U}_{S E_{1}}\left[\rho_{S} \otimes \bigotimes_{i=1}^{n} \rho_{E_{i}}\right],
\end{aligned}
$$

where the initial state is again assumed to be given by Eq. (1). In such a dynamics, the first ancilla $E_{1}$ works as a "memory" in the following sense: The system begins by colliding with $E_{1}$ (memory) according to the map $\mathcal{U}$. The memory then collides with the next ancilla $E_{2}$ via map $\mathcal{V}$, which is followed by a swap $\mathcal{S}$ on $E_{1}$ and $E_{2}$, as shown in Fig. 4(b). The sequence is then iterated, so that the system at each step directly interacts with the memory $E_{1}$ only, while $E_{1}$ collides successively with the remaining ancillas one at a time according to the effective collision map $\mathcal{S}_{1, n} \circ \mathcal{V}_{E_{n} E_{1}}$. We next prove that the open dynamics defined by Eq. (17) coincides with the dynamical map (14).

Using Eq. (16), we can move all swap operators to the left in Eq. (17) and update indexes accordingly as

$$
\begin{aligned}
\rho_{S}^{\prime}(n)= & \operatorname{Tr}_{E_{n} \ldots E_{1}} \mathcal{S}_{1, n} \mathcal{U}_{S E_{n}} \mathcal{S}_{1, n-1} \mathcal{V}_{E_{n} E_{n-1}} \mathcal{U}_{S E_{n-1}} \cdots \\
& \mathcal{S}_{1,2} \mathcal{V}_{E_{3} E_{2}} \mathcal{U}_{S E_{2}} \mathcal{V}_{E_{2} E_{1}} \mathcal{U}_{S E_{1}}\left[\rho_{S} \otimes \bigotimes_{i=1}^{n} \rho_{E_{i}}\right]
\end{aligned}
$$

We next move each partial trace $\operatorname{Tr}_{E_{m}}$ for $2 \leqslant m \leqslant n-1$ to the right until it meets a map acting on $E_{m}$, thus obtaining

$$
\begin{aligned}
\rho_{S}^{\prime}(n)= & \operatorname{Tr}_{E_{n} E_{1}} \mathcal{S}_{1, n} \mathcal{U}_{S E_{n}} \operatorname{Tr}_{E_{n-1}} \mathcal{S}_{1, n-1} \mathcal{V}_{E_{n} E_{n-1}} \mathcal{U}_{S E_{n-1}} \cdots \\
& \operatorname{Tr}_{E_{2}} \mathcal{S}_{1,2} \mathcal{V}_{E_{3} E_{2}} \mathcal{U}_{S E_{2}} \mathcal{V}_{E_{2} E_{1}} \mathcal{U}_{S E_{1}}\left[\rho_{S} \otimes \bigotimes_{i=1}^{n} \rho_{E_{i}}\right]
\end{aligned}
$$

This expression suggests that indeed, to match the exact reduced dynamics at most correlations within a three-qubit system need to be taken into account. With the help of Eq. (15), we can now recursively get rid of the swap operations so as to end up with

$$
\begin{aligned}
\rho_{S}^{\prime}(n)= & \operatorname{Tr}_{E_{n}} \mathcal{U}_{S E_{n}} \operatorname{Tr}_{E_{n-1}} \mathcal{V}_{E_{n} E_{n-1}} \mathcal{U}_{S E_{n-1}} \ldots \\
& \operatorname{Tr}_{E_{2}} \mathcal{V}_{E_{3} E_{2}} \mathcal{U}_{S E_{2}} \operatorname{Tr}_{E_{1}} \mathcal{V}_{E_{2} E_{1}} \mathcal{U}_{S E_{1}}\left[\rho_{S} \otimes \bigotimes_{i=1}^{n} \rho_{E_{i}}\right] \\
= & \operatorname{Tr}_{E_{n} \ldots E_{1}} \mathcal{U}_{S E_{n}} \ldots \mathcal{V}_{E_{2} E_{1}} \mathcal{U}_{S E_{1}}\left[\rho_{S} \otimes \bigotimes_{i=1}^{n} \rho_{E_{i}}\right]
\end{aligned}
$$

which coincides with Eq. (14), and thus identifying $\rho_{S}^{\prime}(n)$ and $\rho_{S}(n)$. This concludes the proof.

The dynamical map $\Lambda(n)$ in Eq. (17), which describes the open dynamics of $S$, thus admits the equivalent representation (12). Note that the above equivalence holds regardless of the form of all the involved collision maps, the initial states of the ancillas, as well as the dimensionality of $S$ and ancillas.

As discussed, considering interactions involving a larger number of ancillas (i.e., $d>1$ ), stronger memory effects can 
be featured in the framework of collision models [64]. This situation can also be reformulated considering a memory of higher dimensionality, whose size is associated to the memory depth, acting as mediator between system and environment. To this aim, let us come back to the evolution described by Eq. (13). In this case, one can consider an equivalent dynamics such that $S$ is repeatedly interacting with two environmental ancillas, which mediate the coupling to the environment by undergoing collisions with ancillas. Indeed, in analogy with Eq. (17), the dynamics defined by

$$
\begin{aligned}
\rho_{S}^{\prime \prime \prime}(n)= & \operatorname{Tr}_{E_{2 n} \ldots E_{1}} \mathcal{U}_{S E_{2}} \mathcal{U}_{S E_{1}} \mathcal{S}_{2,2 n} \mathcal{S}_{1,2 n-1} \mathcal{V}_{E_{2 n-1} E_{2} E_{1}} \ldots \\
& \mathcal{S}_{2,6} \mathcal{S}_{1,5} \mathcal{V}_{E_{5} E_{2} E_{1}} \mathcal{U}_{S E_{2}} \mathcal{U}_{S E_{1}} \mathcal{S}_{2,4} \mathcal{S}_{1,3} \mathcal{V}_{E_{3} E_{2} E_{1}} \\
& \times \mathcal{U}_{S E_{2}} \mathcal{U}_{S E_{1}}\left[\rho_{S} \otimes \bigotimes_{i=1}^{2 n} \rho_{E_{i}}\right]
\end{aligned}
$$

can be shown to be equivalent to Eq. (13). Therefore, also for second-order Markovian dynamics described by the maps $\left\{\Lambda^{(2)}(n)\right\}$, one can consider an equivalent representation of the dynamics by means of a memory whose size is determined by the memory depth. A similar approach can be used for arbitrary $d$. At this point, we remark on the requirement for $S$ to interact with the whole memory before the intraenvironment collisions take place. If, in Eq. (4), $d$-ranged AA collisions occurred after every single $S$ - $E_{i}$ collision, it would remain true that the memory depth, i.e., the relevant correlations to the dynamics, would reside within the last $d$ ancillas that the system interacted with. However, such a setting does not allow us to exploit the swap operation to ensure the system only interacts with the same ancillas throughout its entire dynamics.

\section{RELEVANT CORRELATIONS: THERMODYNAMICS}

\section{A. Entropy}

The previous two sections showed that if one aims at describing the, in general, non-Markovian open system dynamics, then only correlations between the system and a bath portion as large as the range of intraenvironment interactions need to be accounted for. One may wonder if this or a suitably adapted property holds in the characterization of the thermodynamical features, a task for which CMs are increasingly used [3].

For the sake of argument, we will refer to a CM featuring only nearest-neighbor AA collisions, corresponding to a firstorder Markovian process as described by Fig. 1, given by Eq. (12), and explicitly considered in Sec. IV. We will focus on the behavior of entropic quantifiers related to irreversible entropy production (as explained shortly) and, in the last part, heat flux.

Let $S_{S}$ be the von Neumann entropy of the system, $S_{S}=$ $-\operatorname{Tr}\left(\rho_{S} \ln \rho_{S}\right)$. Exploiting the properties of the relative entropy, similarly as in Ref. [76], the dynamical change of $S_{S}$ can be expressed in terms of system-environment correlations, changes in the environment's state, as well as heat exchanged between system and environment. Indeed, after some manipulation, the change in the von Neumann entropy of $S$,

$$
\delta S_{S}(n)=S_{S}(n)-S_{S}(0),
$$

can be exactly decomposed as

$$
\begin{aligned}
\delta S_{S}(n)= & \underbrace{I_{S E}(n)-I_{S E}(0)}_{\mathcal{S}_{\text {corr }}} \\
& +\underbrace{S\left[\rho_{E}(n) \mid \rho_{E}(0)\right]-S\left[\rho_{E}(0) \mid \rho_{E}(0)\right]}_{\mathcal{S}_{\text {env }}} \\
& +\underbrace{\operatorname{Tr}_{E}\left[\rho_{E}(n)-\rho_{E}(0)\right] \ln \rho_{E}(0)}_{Q} \\
= & \mathcal{S}_{\text {corr }}+\mathcal{S}_{\text {env }}+Q,
\end{aligned}
$$

where $S(\rho \mid \sigma)=\operatorname{Tr} \rho(\ln \rho-\ln \sigma)$ is the relative entropy. In particular, for $\rho_{E}(0)=e^{-\beta \hat{H}_{E}} / \operatorname{Tr}\left(e^{-\beta \hat{H}_{E}}\right)$, i.e., a Gibbs state, the third heat-like term is given by

$$
Q=-\beta\left(\left\langle\hat{H}_{E}\right\rangle_{n}-\left\langle\hat{H}_{E}\right\rangle_{0}\right) .
$$

In the standard separation of terms of entropy production [75], which calls for a connection to a thermodynamic viewpoint, the contribution $\mathcal{S}_{\text {corr }}+\mathcal{S}_{\text {env }}$ is usually interpreted as the irreversible entropy production. As shown by Eq. (20), this is related to the establishment of system-environment correlations and changes in the environmental state [76]. The third contribution in Eq. (20) embodies instead the reversible entropy production, associated to the heat exchanged with the environment [cf. Eq. (21)]. Note that the sum of the last two contributions can also be seen as (minus) the variation of the environment entropy over the given time interval, namely, $\delta S_{E}(n)$.

To illustrate the behavior of the three entropic terms in Eq. (20) and how they combine to give the entropy change of $S$ in Eq. (19), let us first consider a greatly simplified collision model, where the environment consists of only two qubits which the system collides with sequentially and iteratively. Thus, the situation is similar to Fig. 1 if we restrict the environment to consist of only ancillas $E_{1}$ and $E_{2}$ which $S$ iteratively interacts with, neither of which is ever traced over nor any correlations shared between the three qubits is ever discarded. In Fig. 5(a), we show the change in entropy evaluated using Eqs. (19) and (20), where we have arbitrarily fixed the initial state of the environment ancillas to be in their respective ground states and assume the partial-SWAP operation for the collision interactions. The three components of Eq. (20) are plotted separately in Fig. 5(b), showing in particular that $\mathcal{S}_{\text {corr }}$ and $\mathcal{S}_{\text {env }}$ are strictly positive, in line with their association to the irreversible entropy production. As expected, the sum of $\mathcal{S}_{\text {corr }}(n), \mathcal{S}_{\text {env }}(n)$, and $Q(n)$ matches $\delta S_{S}(n)$.

Now consider the non-Markovian CM of Fig. 1 with nearest-neighbor AA collisions which, as exhaustively demonstrated in Secs. IV and V, showed that retaining correlations between $S$ and two ancillas at each step is enough to fully capture the open dynamics and, in particular, the degree of non-Markovianity. In light of Fig. 5, one can thus wonder whether by replacing the entire environment with only the few relevant ancillas to the dynamics at each step in Eq. (20), the system's entropy change given by Eq. (19) is again retrieved. This does not occur (not even approximately), as shown in Fig. 6(a) where we compare the behavior of $\delta S_{S}(n)$ and (20) for the erasure scheme B (lower black line), where two ancillas and their correlations are stored at each step, and scheme C (dashed red line), where three ancillas are retained at each 
(a)

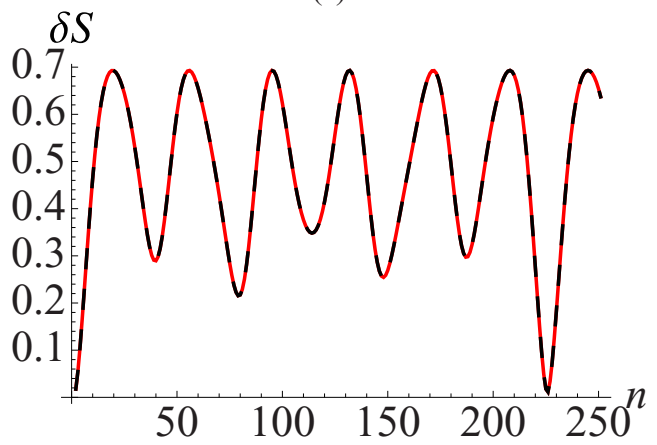

(b)

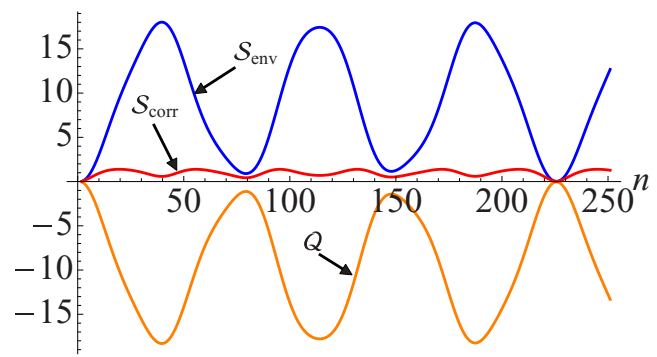

FIG. 5. (a) Simplified CM featuring only two ancillas (with which $S$ interacts successively and iteratively): change in entropy, $\delta S_{S}$, against the number of steps $n$, determined using Eq. (19) (solid colored line) and Eq. (20) (dashed black line). We fix the $S$ initial state to be $|1\rangle$ and the ancillas in $|0\rangle$. (b) We plot the three different contributions entering into Eq. (20). We assume all collisions are partial SWAPs with weak $S-E_{n}$ collisions, $J \tau_{S A}=0.05$, and strong AA collisions with $J \tau_{\mathrm{AA}}=0.95 \frac{\pi}{2}$.

step. Clearly, in the very short-term dynamics, the curves align closely; however, large discrepancies quickly emerge. This discrepancy is not restricted to non-Markovian environments, but occurs even if memory effects are fully absent. This can be seen from Fig. 6(b), where AA collisions are switched off so as to retrieve a fully Markovian situation, showing again a large discrepancy between Eqs. (19) and (20). Thus, it is clear that scheme A also exhibits a large discrepancy qualitatively similar to Fig. 6 (results not shown).

These results strongly indicate that while only correlations between the system and a portion of the bath are relevant for the open dynamics, the description of thermodynamical properties generally demands to account for correlations with the entire environment.

\section{B. Heat flux and non-Markovianity}

For other thermodynamic quantities, exceptions to the above general framework may occur for energy-preserving system-environment couplings [49,52], for instance the Heisenberg interaction [i.e., for $J_{x}=J_{y}=J_{z}$ in Eq. (2)]. In such cases, the heat exchanged by the system fulfills $Q_{S}=$ $-Q_{E}$, where $Q_{S}=\operatorname{Tr}\left\{\hat{H}_{S}\left[\rho_{S}(0)-\rho_{S}(n)\right]\right\}$, while

$$
Q_{E}=\sum_{n} \operatorname{Tr}\left[\hat{H}_{E}\left(\rho_{E_{n}}-\tilde{\rho}_{E_{n}}\right)\right] \text {, }
$$

(a)

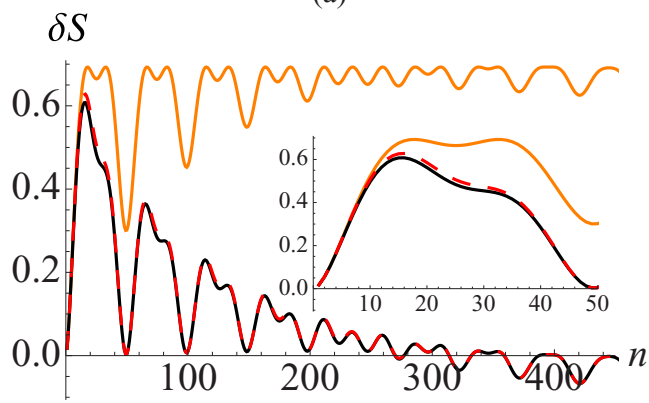

(b)

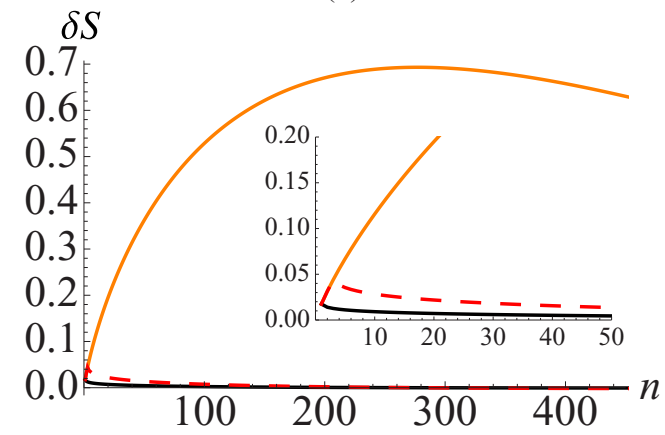

FIG. 6. CM with nearest-neighbor AA collisions: change in entropy $\delta S_{S}(n)$ evaluated using Eq. (19) (top-most, orange line), and using Eq. (20) for erasure schemes B (bottom-most solid black line) and $\mathrm{C}$ (dashed red line) in the (a) case of strong AA collisions with $J \tau_{\mathrm{AA}}=0.95 \frac{\pi}{2}$ and (b) absence of AA collisions. We assume the system is initially in its excited state $|1\rangle$ and the environmental subunits are initially in their ground states. Throughout we assume all collisions are partial SWAPs with weak $S-E_{n}$ collisions, $J \tau_{S A}=0.05$. Insets show the behavior in the first 50 steps.

where $\rho_{E_{n}}\left(\tilde{\rho}_{E_{n}}\right)$ is the state of the $n$th ancilla before (after) it has interacted with the system.

The behavior in time of the heat flux, $\dot{Q}_{S}$, is generally nonmonotonic if memory effects are present $[49,52]$, as shown in a paradigmatic instance in Fig. 7. In the same figure, we plot the behavior of the trace distance for the initial pair of states of $S\{|0\rangle,|1\rangle\}$. In this instance, a relationship emerges between heat flux and the trace distance: their behaviors are perfectly

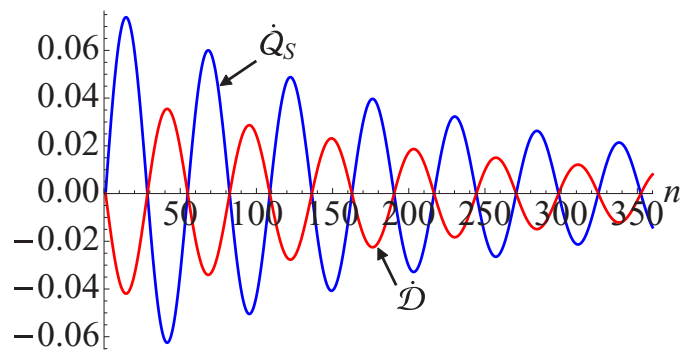

FIG. 7. Behavior of heat flux $\dot{Q}_{S}$ (blue line) and time derivative of the trace distance between the evolution for initial systems states $\{|0\rangle,|1\rangle\}$ (red line). We consider a CM with nearest-neighbor AA partial-SWAP collisions corresponding to $J \tau_{\mathrm{AA}}=0.95 \frac{\pi}{2}$ and weak system-ancilla collisions such that $J \tau_{S A}=0.05$. We assume each ancilla to be initially in a Gibbs state with inverse temperature $\beta=1$. 
aligned with one another, i.e., a nonmonotonic behavior of the heat flux is commensurate with the onset of a non-Markovian dynamics (this can be found with any choice of initial system states except the steady state).

This feature provides a thermodynamic interpretation of non-Markovianity as quantified by information backflow [16] in the case of energy-preserving system-environment interactions. This is because, in such cases, information flow between the system and environment is always accompanied by energy exchange.

\section{CONCLUSIONS}

In this work, through a collision-model-based approach, we investigated the relevance of system-environment correlations in connection with the possibility to embed the system's nonMarkovian dynamics into a Markovian one once the system is extended so as to include a suitably sized bath portion. We considered a simple collision model where the bath is made out of a large collection of ancillas, which the system successively collides with. A memory mechanism is enabled by the occurrence of collisions between the environmental ancillas so as to make the system dynamics generally non-Markovian. Such models allow one to study the relevance of correlations by analyzing how the dynamics is affected by the erasure of correlations between the system and environmental ancillas. We reviewed and generalized the results of Ref. [62], showing that only correlations between the system and a bath portion as large as the range of intraenvironmental interactions matter to the open dynamics, in particular the degree of non-Markovianity. Building on this, we presented a general framework supporting an effective description of the open dynamics. This shows, in particular, how to construct a Markovian embedding for the system dynamics depending on the range of intraenvironmental interactions by introducing the notion of memory depth. Additionally, we provided evidence that at variance with open system dynamics, even in a fully Markovian situation and irrespective of the range of intraenvironmental interactions, all system-environment correlations are generally relevant to the description of thermodynamical quantities such as entropy production. Exceptions can, however, occur for energy-preserving system-environment interactions, in which case we showed that one can give a thermodynamical interpretation in terms of heat flux of the well-known non-Markovianity indicator based on information backflow [16].

As our analysis has focused on quantum dynamics described by a certain class of CMs, we finally address the generality of our conclusions. While any Markovian (CP-divisible) open dynamics can be reproduced through a suitably defined CM, it is not as yet known whether the analogous property holds for arbitrary non-Markovian dynamics, although it was shown this is the case in some paradigmatic instances [30,32,69]. Much of the spirit of our study comes from quantum thermodynamics, where CMs are becoming a popular tool to answer conceptual questions that require some knowledge of the bath dynamics. The class of non-Markovian CMs considered here is conceptually significant in that it enjoys at once two usually demanding properties $[28,66]$ : it leads to a reduced master equation for the system that, like the Lindblad master equation, is ensured to be completely positive. However, in sharp contrast to the Lindblad master equation, this master equation is able to capture strong non-Markovian behavior. The work presented here, in particular Sec. V, thus serves as a significant case study. Its formal extension, and in particular of the intuitive notion of memory depth, to more general non-Markovian dynamics, starting from non-Markovian CMs that rely on different memory mechanisms, is a task for future investigations.

\section{ACKNOWLEDGMENT}

We acknowledge support from the EU Collaborative project QuProCS (Grant Agreement No. 641277) and from MIUR under the FFABR 2017 funding.
[1] H.-P Breuer and F. Petruccione, The Theory of Open Quantum Systems (Oxford University Press, Oxford, 2002).

[2] J. Goold, M. Huber, A. Riera, L. del Rio, and P. Skrzypczyk, The role of quantum information in thermodynamics - A topical review, J. Phys. A: Math. Theor. 49, 143001 (2016).

[3] P. Strasberg, G. Schaller, T. Brandes, and M. Esposito, Quantum and Information Thermodynamics: A Unifying Framework Based on Repeated Interactions, Phys. Rev. X 7, 021003 (2017).

[4] H. Spohn, Entropy production for quantum dynamical semigroups, J. Math. Phys. 19, 1227 (1978).

[5] S. Deffner and E. Lutz, Nonequilibrium Entropy Production for Open Quantum Systems, Phys. Rev. Lett. 107, 140404 (2011).

[6] J. P. Santos, L. C. Céleri, G. T. Landi, and M. Paternostro, The role of quantum coherence in non-equilibrium entropy production, arXiv: 1707.08946

[7] J. P. Santos, G. T. Landi, and M. Paternostro, Wigner Entropy Production Rate, Phys. Rev. Lett. 118, 220601 (2017).

[8] B. Bylicka, M. Tukiainen, D. Chruściński, J. Piilo, and S. Maniscalco, Thermodynamic power of non-Markovianity, Sci. Rep. 6, 27989 (2016).
[9] S. Marcantoni, S. Alipour, F. Benatti, R. Floreanini, and A. T. Rezakhani, Entropy production and non-markovian dynamical maps, Sci. Rep. 7, 12447 (2017).

[10] G. Guarnieri, C. Uchiyama, and B. Vacchini, Energy backflow and non-Markovian dynamics, Phys. Rev. A 93, 012118 (2016).

[11] G. Guarnieri, J. Nokkala, R. Schmidt, S. Maniscalco, and B. Vacchini, Energy backflow in strongly coupled non-Markovian continuous-variable systems, Phys. Rev. A 94, 062101 (2016).

[12] S. Bhattacharya, A. Misra, C. Mukhopadhyay, and A. K. Pati, Exact master equation for a spin interacting with a spin bath: Non-Markovianity and negative entropy production rate, Phys. Rev. A 95, 012122 (2017).

[13] M. Popovic, B. Vacchini, and S. Campbell, Entropy production and correlations in a controlled non-Markovian setting, Phys. Rev. A 98, 012130 (2018).

[14] H.-P. Breuer, E.-M. Laine, J. Piilo, and B. Vacchini, Colloquium: Non-Markovian dynamics in open quantum systems, Rev. Mod. Phys. 88, 021002 (2016).

[15] S. Campbell, A. Smirne, L. Mazzola, N. Lo Gullo, B. Vacchini, Th. Busch, and M. Paternostro, Critical assessment of two-qubit 
post-Markovian master equations, Phys. Rev. A 85, 032120 (2012).

[16] H.-P. Breuer, E.-M. Laine, and J. Piilo, Measure for the Degree of Non-Markovian Behavior of Quantum Processes in Open Systems, Phys. Rev. Lett. 103, 210401 (2009).

[17] J. Iles-Smith, N. Lambert, and A. Nazir, Environmental dynamics, correlations, and the emergence of noncanonical equilibrium states in open quantum systems, Phys. Rev. A 90, 032114 (2014).

[18] S. Xue, T. Nguyen, M. R. James, A. Shabani, V. Ugrinovskii, and I. R. Petersen, Modelling and filtering for non-Markovian quantum systems, arXiv:1704.00986.

[19] S. Xue, M. R. Hush, and I. R. Petersen, Feedback tracking control of non-Markovian quantum systems, IEEE Trans. Control Syst. Technol. 25, 1552 (2017).

[20] D. Tamascelli, A. Smirne, S. F. Huelga, and M. B. Plenio, Nonperturbative Treatment of Non-Markovian Dynamics of Open Quantum Systems, Phys. Rev. Lett. 120, 030402 (2018).

[21] J. Rau, Relaxation phenomena in spin and harmonic oscillator systems, Phys. Rev. 129, 1880 (1963).

[22] T. A. Brun, A simple model of quantum trajectories, Am. J. Phys. 70, 719 (2002).

[23] V. Scarani, M. Ziman, P. Štelmachovič, N. Gisin, and V. Bužek, Thermalizing Quantum Machines: Dissipation and Entanglement, Phys. Rev. Lett. 88, 097905 (2002).

[24] M. Ziman and V. Bužek, All (qubit) decoherences: Complete characterization and physical implementation, Phys. Rev. A 72, 022110 (2005).

[25] V. Giovannetti and G. M. Palma, Master Equations for Correlated Quantum Channels, Phys. Rev. Lett. 108, 040401 (2012).

[26] K. Modi, C. A. Rodríguez-Rosario, and A. Aspuru-Guzik, Positivity in the presence of initial system-environment correlation, Phys. Rev. A 86, 064102 (2012).

[27] T. Rybár, S. N. Filippov, M. Ziman, and V. Bužek, Simulation of indivisible qubit channels in collision models, J. Phys. B 45, 154006 (2012).

[28] F. Ciccarello, G. M. Palma, and V. Giovannetti, Collision-modelbased approach to non-Markovian quantum dynamics, Phys. Rev. A 87, 040103 (2013).

[29] N. K. Bernardes, A. R. R. Carvalho, C. H. Monken, and M. F. Santos, Environmental correlations and markovian to nonmarkovian transitions in collisional models, Phys. Rev. A 90, 032111 (2014).

[30] S. Kretschmer, K. Luoma, and W. T. Strunz, Collision model for non-Markovian quantum dynamics, Phys. Rev. A 94, 012106 (2016).

[31] S. Lorenzo, F. Ciccarello, and G. M. Palma, Class of exact memory-kernel master equations, Phys. Rev. A 93, 052111 (2016).

[32] S. Lorenzo, F. Ciccarello, and G. M. Palma, Composite quantum collision models, Phys. Rev. A 96, 032107 (2017).

[33] S. N. Filippov, J. Piilo, S. Maniscalco, and M. Ziman, Divisibility of quantum dynamical maps and collision models, Phys. Rev. A 96, 032111 (2017).

[34] A. Bodor, L. Diósi, Z. Kallus, and T. Konrad, Structural features of non-Markovian open quantum systems using quantum chains, Phys. Rev. A 87, 052113 (2013).

[35] E. Mascarenhas and I. de Vega, Quantum critical probing and simulation of colored quantum noise, Phys. Rev. A 96, 062117 (2017).
[36] N. K. Bernardes, A. R. R. Carvalho, C. H. Monken, and M. F. Santos, Coarse graining a non-Markovian collisional model, Phys. Rev. A 95, 032117 (2017).

[37] J. Jin and C.-S. Yu, Non-Markovianity in a collision model with environmental block, New J. Phys. 20, 053026 (2018).

[38] Z.-X. Man, Y.-J. Xia, and R. Lo Franco, Temperature effects on quantum non-Markovianity via collision models, Phys. Rev. A 97, 062104 (2018).

[39] A. L. Grimsmo, Time-Delayed Quantum Feedback Control, Phys. Rev. Lett. 115, 060402 (2015).

[40] H. Pichler and P. Zoller, Photonic Circuits with Time Delays and Quantum Feedback, Phys. Rev. Lett. 116, 093601 (2016).

[41] J. A. Gross, C. M. Caves, G. J. Milburn, and J. Combes, Qubit models of weak continuous measurements: Markovian conditional and open-system dynamics, Quantum Sci. Technol. 3, 024005 (2018).

[42] F. Ciccarello, Collision models in quantum optics, Quantum Meas. Quantum Metrol. 4, 53 (2017).

[43] D. Kafri, J. M. Taylor, and G. J. Milburn, A classical channel model for gravitational decoherence, New J. Phys. 16, 065020 (2014).

[44] N. Altamirano, P. Corona-Ugalde, R. B. Mann, and M. Zych, Unitarity, feedback, interactions-Dynamics emergent from repeated measurements, New J. Phys. 19, 013035 (2017).

[45] B. Sutton and S. Datta, Manipulating quantum information with spin torque, Sci. Rep. 5, 17912 (2015).

[46] D. Layden, E. Martín-Martínez, and A. Kempf, Universal scheme for indirect quantum control, Phys. Rev. A 93, 040301 (2016).

[47] K. Beyer, K. Luoma, and W. T. Strunz, Collision-model approach to steering of an open driven qubit, Phys. Rev. A 97, 032113 (2018).

[48] B. Çakmak, S. Campbell, B. Vacchini, Ö. E. Müstecaplığlu, and M. Paternostro, Robust multipartite entanglement generation via cascaded interactions, arXiv:1803.05243.

[49] S. Lorenzo, A. Farace, F. Ciccarello, G. M. Palma, and V. Giovannetti, Heat flux and quantum correlations in dissipative cascaded systems, Phys. Rev. A 91, 022121 (2015).

[50] H.-B. Chen, G.-Y. Chen, and Y.-N. Chen, Thermodynamic description of non-Markovian information flux of nonequilibrium open quantum systems, Phys. Rev. A 96, 062114 (2017).

[51] M. Pezzutto, M. Paternostro, and Y. Omar, Implications of nonMarkovian quantum dynamics for the Landauer bound, New J. Phys. 18, 123018 (2016).

[52] S. Lorenzo, R. McCloskey, F. Ciccarello, M. Paternostro, and G. M. Palma, Landauer's Principle in Multipartite Open Quantum System Dynamics, Phys. Rev. Lett. 115, 120403 (2015).

[53] L. Diosi, T. Feldmann, and R. Kosloff, On the exact identity between thermodynamics and informatic entropies in a unitary model of friction, Int. J. Quantum Inf. 4, 99 (2006).

[54] E. Bäumer, M. Perarnau-Llobet, P. Kammerlander, and R. Renner, Partial thermalizations allow for optimal thermodynamic processes, arXiv:1712.07128.

[55] E. Pereira, Heat, work, and energy currents in the boundarydriven $x x z$ spin chain, Phys. Rev. E 97, 022115 (2018).

[56] L. Li, J. Zou, H. Li, B.-M. Xu, Y.-M. Wang, and B. Shao, Effect of coherence of nonthermal reservoirs on heat transport in a microscopic collision model, Phys. Rev. E 97, 022111 (2018). 
[57] B. Çakmak, A. Manatuly, and Ö. E. Müstecaplığlu, Thermal production, protection, and heat exchange of quantum coherences, Phys. Rev. A 96, 032117 (2017).

[58] F. Barra, The thermodynamic cost of driving quantum systems by their boundaries, Sci. Rep. 5, 14873 (2015).

[59] R. Uzdin and R. Kosloff, The multilevel four-stroke swap engine and its environment, New J. Phys. 16, 095003 (2014).

[60] J. M. Horowitz and J. M. R. Parrondo, Entropy production along nonequilibrium quantum jump trajectories, New J. Phys. 15, 085028 (2013).

[61] J. M. Horowitz, Quantum-trajectory approach to the stochastic thermodynamics of a forced harmonic oscillator, Phys. Rev. E 85, 031110 (2012).

[62] R. McCloskey and M. Paternostro, Non-Markovianity and system-environment correlations in a microscopic collision model, Phys. Rev. A 89, 052120 (2014).

[63] P. Siegle, I. Goychuk, P. Talkner, and P. Hänggi, Markovian embedding of non-Markovian superdiffusion, Phys. Rev. E 81, 011136 (2010).

[64] B. Çakmak, M. Pezzutto, M. Paternostro, and Ö. E. Müstecaplığlu, Non-Markovianity, coherence, and systemenvironment correlations in a long-range collision model, Phys. Rev. A 96, 022109 (2017).

[65] F. Ciccarello and V. Giovannetti, A quantum non-Markovian collision model: Incoherent swap case, Phys. Scr. 2013, 014010 (2013).
[66] B. Vacchini, Non-Markovian master equations from piecewise dynamics, Phys. Rev. A 87, 030101 (2013).

[67] B. Vacchini, General structure of quantum collisional models, Int. J. Quantum Inf. 12, 1461011 (2014).

[68] B. Vacchini, Generalized Master Equations Leading to Completely Positive Dynamics, Phys. Rev. Lett. 117, 230401 (2016).

[69] S. Lorenzo, F. Ciccarello, G. M. Palma, and B. Vacchini, Quantum non-Markovian piecewise dynamics from collision models, Open Syst. Inf. Dyn. 24, 1740011 (2017).

[70] D. Chruściński and A. Kossakowski, Sufficient conditions for a memory-kernel master equation, Phys. Rev. A 94, 020103 (2016).

[71] D. Chruściński and A. Kossakowski, Generalized semi-Markov quantum evolution, Phys. Rev. A 95, 042131 (2017).

[72] M. A. Nielsen and I. L. Chuang, Quantum Computation and Quantum Information (Cambridge University Press, Cambridge, 2000).

[73] S. Wißmann, A. Karlsson, E.-M. Laine, J. Piilo, and H.-P. Breuer, Optimal state pairs for non-Markovian quantum dynamics, Phys. Rev. A 86, 062108 (2012).

[74] W.-K. Ching and M. K. Ng, Markov Chains: Models, Algorithms and Applications (Springer, New York, 2006) .

[75] S. R. de Groot and P. Mazur, Non-Equilibrium Thermodynamics (Dover, New York, 1984) .

[76] M. Esposito, K. Lindenberg, and C. Van den Broeck, Entropy production as correlation between system and reservoir, New J. Phys. 12, 013013 (2010). 